\title{
Erratum to: Fungal diversity notes 111-252-taxonomic and phylogenetic contributions to fungal taxa
}

\author{
Hiran A. Ariyawansa ${ }^{1,3} \cdot$ Kevin D. Hyde ${ }^{1,2,3,15} \cdot$ Subashini C. Jayasiri $^{1,3} \cdot$ Bart Buyck $^{4}$. \\ K. W. Thilini Chethana ${ }^{3,5}$ • Dong Qin Dai ${ }^{2,3}$ - Yu Cheng Dai ${ }^{8}$. \\ Dinushani A. Daranagama $^{3,9}$ • Ruvishika S. Jayawardena ${ }^{3,5} \cdot$ Robert Lücking $^{11,47}$.

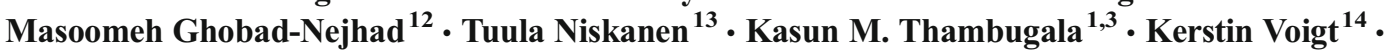 \\ Rui Lin Zhao ${ }^{9}$ - Guo-Jie Li ${ }^{9}$ - Mingkwan Doilom ${ }^{2,3}$ - Saranyaphat Boonmee $^{3}$ - Zhu L. Yang ${ }^{2}$. \\ Qing Cai ${ }^{2}$ - Yang-Yang Cui ${ }^{2}$ Ali H. Bahkali ${ }^{15}$. Jie Chen ${ }^{3,8} \cdot$ Bao Kai Cui $^{8}$ • Jia Jia Chen ${ }^{8}$ Monika C. Dayarathne ${ }^{2,3}$. \\ Asha J. Dissanayake ${ }^{3,5}$ - Anusha H. Ekanayaka ${ }^{3}$ - Akira Hashimoto ${ }^{16,17}$ • Sinang Hongsanan ${ }^{2,3}$. \\ E. B. Gareth Jones ${ }^{15}$ • Ellen Larsson ${ }^{18}$ • Wen Jing $\mathrm{Li}^{3}$ • Qi-Rui Li ${ }^{3}$ Jian Kui Liu ${ }^{1,3}$. \\ Zong Long Luo ${ }^{20}$ - Sajeewa S. N. Maharachchikumbura ${ }^{1,3}$ - Ausana Mapook ${ }^{2,3}$. \\ Eric H. C. McKenzie ${ }^{21}$ - Chada Norphanphoun ${ }^{3}$ - Sirinapa Konta ${ }^{3}$ - Ka Lai Pang ${ }^{19}$. \\ Rekhani H. Perera ${ }^{2,3}$ • Rungtiwa Phookamsak ${ }^{2,3}$ • Chayanard Phukhamsakda ${ }^{3}$. \\ Umpava Pinruan $^{49}$ - Emile Randrianjohany ${ }^{22}$ - Chonticha Singtripop ${ }^{2,3}$ - Kazuaki Tanaka ${ }^{17}$. \\ Cheng Ming $\operatorname{Tian}^{23} \cdot$ Saowaluck Tibpromma ${ }^{2,3}$ - Mohamed A. Abdel-Wahab ${ }^{15,27}$. \\ Dhanushka N. Wanasinghe ${ }^{2,3}$ • Nalin N. Wijayawardene ${ }^{3,25}$ • Jin-Feng Zhang ${ }^{3}$. \\ Huang Zhang ${ }^{26}$ • Faten A. Abdel-Aziz ${ }^{27}$ - Mats Wedin ${ }^{28}$ - Martin Westberg ${ }^{28}$. \\ Joseph F. Ammirati ${ }^{29}$ - Timur S. Bulgakov ${ }^{30}$ • Diogo X. Lima ${ }^{31}$ • Tony M. Callaghan ${ }^{32}$. \\ Philipp Callac $^{33}$ - Cheng-Hao Chang ${ }^{34}$ - Luis F. Coca ${ }^{35}$ - Manuela Dal-Forno ${ }^{36}$ • \\ Veronika Dollhofer $^{37}$ • Kateřina Fliegerová ${ }^{38}$ - Katrin Greiner ${ }^{39}$ - Gareth W. Griffith $^{32}$. \\ Hsiao-Man $\mathrm{Ho}^{34}$ • Valerie Hofstetter ${ }^{40}$ • Rajesh Jeewon ${ }^{41}$ • Ji Chuan Kang ${ }^{42}$ • \\ Ting-Chi Wen ${ }^{42}$ • Paul M. Kirk ${ }^{43}$ • Ilkka Kytövuori ${ }^{44}$ • James D. Lawrey ${ }^{45}$ • Jia Xing ${ }^{5}$. \\ Hong $\mathrm{Li}^{5}$ • Zou Yi Liu ${ }^{1}$ - Xing Zhong Liu ${ }^{9}$ - Kare Liimatainen ${ }^{46} \cdot$ H. Thorsten Lumbsch ${ }^{47}$. \\ Misato Matsumura $^{17} \cdot$ Bibiana Moncada $^{48} \cdot$ Salilaporn Nuankaew $^{49} \cdot$ Sittiporn Parnmen $^{50}$. $^{\circ}$ \\ André L. C. M. de Azevedo Santiago ${ }^{31}$ • Sujinda Sommai ${ }^{49}$ - Yu Song ${ }^{2}$ Carlos A. F. de Souza ${ }^{31}$. \\ Cristina M. de Souza-Motta ${ }^{31}$ • Hong Yan $\mathrm{Su}^{20}$ • Satinee Suetrong ${ }^{51}$ • Yong Wang ${ }^{25}$. \\ Syuan-Fong Wei ${ }^{34}$ • Ting Chi Wen ${ }^{25}$. Hai Sheng Yuan ${ }^{52}$ - Li Wei Zhou ${ }^{52}$.

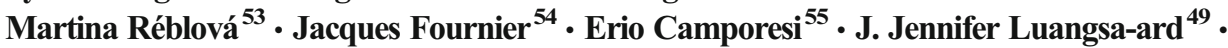 \\ Kanoksri Tasanathai $^{49}$ - Artit Khonsanit ${ }^{49}$ - Donnaya Thanakitpipattana ${ }^{49}$ • Sayanh Somrithipol $^{49}$. \\ Paul Diederich $^{57}$ - Ana M. Millanes ${ }^{56} \cdot$ Ralph S. Common $^{58} \cdot$ Marc Stadler $^{6}$ • Ji Ye Yan $^{5}$. \\ XingHong $\mathrm{Li}^{5}$ • Hye Won Lee ${ }^{24}$. Thi T. T. Nguyen ${ }^{24}$ • Hyang Burm Lee ${ }^{24}$ Eliseo Battistin ${ }^{59}$. \\ Orlando Marsico $^{62}$ - Alfredo Vizzini ${ }^{61}$ - Jordi Vila ${ }^{63}$ • Enrico Ercole ${ }^{61}$ - Ursula Eberhardt ${ }^{7}$ Giampaolo Simonini $^{60}$ . $^{6}$ \\ Hua-An Wen ${ }^{9}$ Xin-Hua Chen ${ }^{64}$ - Otto Miettinen ${ }^{65}$ • Viacheslav Spirin ${ }^{65}$ - Hernawati ${ }^{10}$
}

Published online: 3 December 2015

(C) School of Science 2015

The online version of the original article can be found at http://dx.doi.org/ 10.1007/s13225-015-0346-5.

Zou Yi Liu

gzliuzuoyi@163.com

Guizhou Key Laboratory of Agricultural Biotechnology, Guizhou Academy of Agricultural Sciences, Guiyang 550006, Guizhou, China
Key Laboratory for Plant Diversity and Biogeography of East Asia, Kunming Institute of Botany, Chinese Academy of Science, Kunming 650201, Yunnan, China

Centre of Excellence in Fungal Research, Mae Fah Luang University, Chiang Rai 57100, Thailand 


\section{Erratum to: Fungal Diversity DOI 10.1007/s13225-015-0346-5}

In the initial online publication, three authors were omitted and some authors' affiliations were incorrect. The online publication has been updated to address these errors. The authors and affiliations listed in this erratum are the same as in the updated article.

$4 \quad$ Département Systématique et Evolution, CP 39, ISYEB, UMR 7205 CNRS MNHN UPMC EPHE, Muséum National d'Histoire Naturelle, 12 Rue Buffon, F-75005 Paris, France

5 Institute of Plant and Environment Protection, Beijing Academy of Agriculture and Forestry Sciences, No. 9 of Shu Guang Hua Yuan Zhong Lu, Haidian District, Beijing 10097, China

6 Microbial Drugs Helmholtz-Centre for Infection Research, Inhoffenstrasse 7, 38124 Braunschweig, Germany

7 Staatliches Museum für Naturkunde Stuttgart, Rosenstein 1, D-70191 Stuttgart, Germany

8 Institute of Microbiology, Beijing Forestry University, PO Box 61, Beijing 100083, China

9 State Key Laboratory of Mycology, Institute of Microbiology, Chinese Academy of Sciences, No 3 1st West Beichen Road, Chaoyang District, Beijing 100101, China

10 The Herbarium (ANDA), Universitas Andalas, Padang, Sumatera Barat 25163, Indonesia

11 Botanischer Garten \& Botanisches Museum Berlin, Freie Universität Berlin, Königin-Luise-Straße 6-8, 14195 Berlin, Germany

12 Department of Biotechnology, Iranian Research Organization for Science and Technology (IROST), P. O. Box 3353-5111, Tehran 3353136846, Iran

13 Jodrell Laboratory, Royal Botanic Gardens, Kew TW9 3DS, UK

14 Leibniz Institute for Natural Product Research and Infection Biology, Jena Microbial Resource Collection, Adolf-Reichwein-Strasse 23, 07745 Jena, Germany

15 Department of Botany and Microbiology, College of Science, King Saud University, P.O. Box: 2455, Riyadh 1145, Saudi Arabia

16 The United Graduate School of Agricultural Sciences, Iwate University, 18-8 Ueda 3 Chome, Morioka 020-8550, Japan

17 Faculty of Agriculture and Life Sciences, Hirosaki University, 3 Bunkyo-cho, Hirosaki, Aomori 036-8561, Japan

18 Department of Biological and Environmental Sciences, University of Gothenburg, Box 461, SE-40530 Göteborg, Sweden

19 Institute of Marine Biology and Center of Excellence for the Oceans, National Taiwan Ocean University, 2 Pei-Ning Road,

Keelung 20224, Taiwan, Republic of China

20 College of Agriculture \& Biology, Dali University, Dali, 671003 Yunnan, People's Republic of China

21 Manaaki Whenua Landcare Research, Private Bag 92170, Auckland, New Zealand
22 Centre National de Recherchesurl' Environnement (CNRE), BP 1739, Lab. de Microbiologie de l'Environnement (LME), Antananarivo, Madagascar

23 The Key Laboratory for Silviculture and Conservation of Ministry of Education, Beijing Forestry University, Beijing 100083, China

24 Division of Food Technology, Biotechnology \& Agrochemistry, College of Agriculture \& Life Sciences, Chonnam National University, Gwangju 61186, South Korea

25 Department of Plant Pathology, Agriculture College, Guizhou University, Guizhou 550025, People's Republic of China

26 Yunnan Institute of Food Safety, Kunming University of Science \& Technology, Kunming 650500, People's Republic of China

27 Department of Botany, Faculty of Science, Sohag University, Sohag, Egypt

28 Department of Botany, Swedish Museum of Natural History, PO Box 50007, SE-10405 Stockholm, Sweden

29 Department of Biology, University of Washington, 351800, Seattle, WA 98195, USA

30 Academy of Biology and Biotechnology, Southern Federal University, Rostov-on-Don 344090, Rostov Region, Russia

31 Programa de Pós Graduação em Biologia de Fungos, Departamento de Micologia, Universidade Federal de Pernambuco, Av. Prof. Nelson Chaves, s/n, 50670-420 Recife, PE, Brazil

32 Institute of Biological, Environmental and Rural Sciences, Aberystwyth University, Adeilad Cledwyn, Penglais, Aberystwyth, Ceredigion SY23 3DD, UK

33 INRA, UR1264, MycSA, BP81, F-33883 Villenave d'Ornon, France

34 Department of Science Education, National Taipei University of Education, 134, Sect. 2, Heping E. Rd., Taipei, Taiwan

35 Herbario Universidad de Caldas (FAUC), Edificio Bicentenario, Calle 65, No. 26-10, Manizales A.A. 275, Caldas, Colombia

36 Department of Environmental Science and Policy, George Mason University, Fairfax, VA 22030-4444, USA

37 Bavarian State Research Center for Agriculture, Central Department for Quality Assurance and Analytics, Micro- and Molecular Biology, Lange Point, 685354 Freising, Germany

38 Institute of Animal Physiology and Genetics, Czech Academy of Sciences, v.v.i., Videnska 1083, 14220 Prague, Czech Republic

39 Department of Mycology, University of Bayreuth, Universitätsstraße 30, 95440 Bayreuth, Germany

40 Department of Plant Protection, Agroscope Changins-Wadenswil Research Station ACW, Rte de Duiller, 1260 Nyon, Switzerland 
$\overline{41}$ Department of Health Sciences, Faculty of Science, University of Mauritius, Reduit, Mauritius

42 The Engineering and Research Center for Southwest Bio-Pharmaceutical Resources of National Education Ministry of China, Guizhou University, Guiyang 550025, Guizhou Province, China

43 Royal Botanic Gardens, Kew, Richmond, Surrey TW9 3DS, UK

44 Botanical Museum, University of Helsinki, Helsinki, P.O. Box 7, 00014, Finland

45 Department of Biology, George Mason University, Fairfax, VA 22030-4444, USA

46 Plant Biology, Department of Biosciences, University of Helsinki, Helsinki, P.O. Box 65, 00014, Finland

47 Science \& Education, The Field Museum, 1400 South Lake Shore Drive, Chicago, IL 60605, USA

48 Universidad Distrital Francisco José de Caldas, Cra. 4 No. 26B-54, Torre de Laboratorios, Herbario, Bogotá, Colombia

49 Microbe Interaction Laboratory (BMIT), BIOTEC, National Science and Technology Development Agency (NSTDA), 113 Thailand Science Park, Thanon Phahonyothin, Tombon Khlong Nueng, Amphoe, Khlong Luang, Pathum Thani 12120, Thailand

50 Toxicology and Biochemistry Section, Department of Medical Sciences, Ministry of Public Health, Nonthaburi 11000, Thailand

51 Fungal Biodiversity Laboratory, BIOTEC,

National Science and Technology Development Agency (NSTDA), 113 Thailand Science Park, Thanon Phahonyothin,
Tombon Khlong Nueng, Amphoe Khlong Luang, PathumThani 12120, Thailand

52 Institute of Applied Ecology, Chinese Academy of Sciences, Shenyang 110164, China

53 Department of Taxonomy, Institute of Botany of the Academy of Sciences, Průhonice, Czech Republic

54 Las Muros, Rimont 09420, France

55 A.M.B. Gruppo Micologico Forlivese "Antonio Cicognani”, Via Roma 18, Forlì, Italy

56 Departamento de Biología y Geología, Física y Química Inorgánica, Universidad Rey Juan Carlos, E-28933 Móstoles, Spain

57 Musée national d'histoire naturelle, 25 rue Munster, L-2160 Luxembourg, Luxembourg

58534 Fenton St, Lansing, MI 48910, USA

59 Natural History Museum, Corso Italia 63, 36078 Valdagno, Italy

60 Via Bell'aria 8, I-42121 Reggio Emilia, Italy

61 Department of Life Sciences and Systems Biology, University of Torino, Viale P.A. Mattioli 25, 10125 Torino, Italy

62 Via Panoramica - I-87047, San Pietro in Guarano (CS), Italy

63 PO Box 30041, E-08034, Barcelona, Spain

64 Faculty of Basic Medical Sciences, Guilin Medical University, Guilin 541004, People's Republic of China

65 Finnish Museum of Natural History, Botanical Museum, University of Helsinki, Helsinki, PO Box 7, 00014, Finland 